\title{
Active Intelligent Vision Using the Dynamic Generalized Hough Transform
}

\author{
V. F. Leavers
}

Physics Dept, King's College
Strand, London WC2R 2LS

Parametric transformation is a powerful tool in shape analysis which gives good results even in the presence of noise and occlusion. Major shortcomings of the technique are excessive storage requirements, computational complexity and the need to initiate a separate transformation process with respect to each feature under detection. In addition, standard parametric transformation processes the entire image treating each image point independently. The proposed method selectively segments the image and provides a feedback mechanism linking image and transform space. Decisions are made concerning the probable instance of shape under detection, the viability of processing and the need to gather further evidence from the image. The processing monitors itself and adapts to focus on areas of interest avoiding computationally expensive global processing. Low level processing and high level decision making are provided with a window of communication not previously available using such parametric transform methods. The method is applied and illustrated with respect to the detection of circles and elipses.

The Hough transform[1] works by grouping low level feature points (edge image data) into object specific intermediate features (e.g. line segments). This is accomplished using the low level feature points to generate information concerning all possible groupings of points within the image. The corresponding transform plane is the accumulation of that evidence. The technique is computationally intensive because evidence is generated of all possible groupings of points in the image.

Previous suggested approaches may be divided into three categories. The first seeks to reduce the computational load by using information from the image to reduce the generation of evidence in the transform plane[2], [3], [4], [5]. The second class of methods involves absolute or iterative reductions in resolution of either the transform or the image space[6], [7], [8], [9]. Where more than two parameters are under detection the

method may involve impractically large computation and storage requirements. To deal with this particular difficulty, a third class of methods decomposes the computation into separate stages[10], [11], [12], [13], each stage passing results to the next. The present method, the DGHT[14], $[15],[16]$ uses information available in the relative distribution of feature points to optimise the computation of the transform. The $n$ parameters associated with the shape under detection are calculated using a single fixed image point and all possible combinations of that image point with sets of $(n-1)$ other image points. Each parameter is calculated independently and requires only one dimensional accumulation of evidence. Hence if $T$ is the resolution in transform space and $n$ is the number of parameters under detection then use of the DGHT reduces memory requirements from $T^{n}$ to $n T$ and introduces the opportunity for parallel calculation and accumulation of parameters.

Peaks in the histograms will indicate the parameters associated with the most probable instance of the shape in image space of which the fixed point is a member. This information may be used to detect that shape and to remove the points associated with it from the image. The process is then repeated using the shortened list. Peak detection in the transform space normally presents difficulties. The combinatorial nature of the generation of peaks using the DGHT means that peaks are significantly higher than the background and detection is one dimensional.

Global processing of the image has remained a standard feature of parametric transformation. The DGHT is sensitive to feature point density in the image and it is therefore expedient to segment the image. Uniform segmentation has been used previously[15] for a simple image of a single object. Where the image contains multiple objects or occlusion is present, segmentation cannot be fixed but needs to be adapted to the image and the type of shape under detection. The DGHT provides a feedback mechanism linking image and transform space. Decisions can be made concerning the probable instance of shape under detection, 
the viability of processing and the need to gather further evidence from the image. This means that the processing can monitor itself and adapt to focus on areas of interest avoiding computationally expensive global processing. The present work explores this requirement.

\section{The Dynamic Generalized Hough transform}

An expression for the Generalized Hough Transform, GHT, may be written in the form suggested by Deans[1]

$$
f(\xi, p)=\iint_{D} F(x, y) \delta(p-C(x, y ; \xi)) d x d y
$$

where $F(x, y)$ is an arbitrary generalized function[2] defined on the $x y$ plane $D$ and the argument of the delta function defines some family of curves in the xy plane parametrized by the scalar $p$ and the components $\xi_{1}, \xi_{2}, \ldots \xi_{n}$ of the vector $\xi$.

If, $F(x, y)$, represents a binary image the integral of equation 1 will have a value of 1 when the argument of the delta function evaluates to zero. in computational terms, this occurs at all values of $(x, y)$ that are solutions to the discrete equation

$$
p_{j}=C\left(x_{i}, y_{i} ; \xi_{j}\right)
$$

and this equation is used to calculate the standard GHT. The $i, j$ subscripts refer to ordered sets in the image and the transform space respectively. For every point, $\left(x_{i}, y_{i}\right)$, of the image, $i$ is fixed and the values $p_{j}$ are calculated using stepwise increments of the components of $\xi_{j}$. Each point, $\left(p_{j}, \xi_{j}\right)$, in the transform space will refer to a possible curve in image space which passes through the point $\left(x_{i}, y_{i}\right)$. The SHT therefore provides a great redundancy of information concerning the image. This is because each image point is treated independently.

The DGHT proposes that image points are tested for the most probable, as opposed to all possible, membership of a shape. (In two dimensions shape may refer to a curve and in three dimensions a surface). If, when the image is scanned for candidate feature points, a list of those feature points is maintained, then possible membership of curves/surfaces may be tested. Where $n$ parameters are associated with the shape under detection then a minimum of $n$ points are required to test the membership of a shape of any given point and any other $(n-1)$ image points. What follows is an application to the detection of conic sections, in particular circles and ellipses.

\section{Active Intelligent Detection of Conic Sections.}

The general equation of the conic section is:

$$
a x^{2}+h x y+b y^{2}+2 g x+2 f y+c=0
$$

In the case of the circle, $a=b$. The number of parameters may then be reduced to three by normalisation. For an ellipse equation 3 has one parameter too many. For a well determined model a constant needs to be applied to $a, b, h, g, f$ and c. This needs to be done in a manner that leads to a numerically stable model. It can be shown[3] that an appropriate constraint for the detection of ellipses is to set $a+c=$ Constant. The equation can now be written:

$$
x^{2}+y^{2}-U\left(x^{2}-y^{2}\right)-V 2 x y-R x-S y-T=0
$$

The ellipse eccentricity is given by $e=b / a$ and

$$
\begin{gathered}
U=\cos 2 \alpha \frac{1-e^{2}}{1+e^{2}} \\
V=\sin 2 \alpha \frac{1-e^{2}}{1+e^{2}} \\
R=2 x_{0}(1-U)-2 y_{0} V \\
S=2 y_{0}(1+U)-2 x_{0} V \\
T=\frac{2 a^{2} b^{2}}{a^{2}+b^{2}}-\frac{x_{0} R}{2}-\frac{y_{0} S}{2}
\end{gathered}
$$

$U$ and $V$ depend only on $\alpha$, the angle of rotation of the ellipse and $e$, its eccentricity. In particular, for a circle, $U$ and $V$ are zero. In the case of images which may contain both circles and ellipses such a mixture may be detected with a single scan of the image. Equation 4 is used to deduce the eigenvalues of each combination of image points. The eigenvector corresponding to the least eigenvalue will contain the parameters under detection[3].

Processing begins by scanning the edge image for feature points. When a likely point is encountered a simple check is made to eliminate isolated noise points. Points around the candidate first point are summed first in a horizontal direction and then a vertical direction. If either sum exceeds a threshold then this point is accepted.

The efficiency of the DGHT is sensitive to feature point density in the image. It is therefore necessary to segment the image. Having selected a candidate first feature point the algorithm focuses on an area of interest around that point using a 
fixed size of segment. The results of the transformation of the feature points contained in that segment are then tested. Where a calculated parameter has a percentage contribution to a maximum value of less than a predetermined percentage of feature points processed then this is an unreliable result requiring further accumulation of evidence. The processing thus adjusts the size of the segment and continues the transformation and accumulation process. For the circle center co-ordinates and radius respectively, $x_{0}(30 \%), y_{0}(30 \%), r(30 \%)$ are acceptable percentages. For the ellipse center co-ordinates, major and minor axes and angle of rotation respectively, $x_{0}(20 \%), y_{0}(20 \%), a(15 \%)$, $b(15 \%), \alpha(10 \%)$ are acceptable percentages. It is not within the scope of such a short paper to discuss the data dependence of these thresholds, they are simply stated. It should be noted that the algorithm allows parallel calculation and accumulation of the parameters.

\section{Computational Complexity}

If $N$ is the number of parameters under detection and $m$ is the number of features points in a segment then the number of operations associated with the detection of either a circle or an ellipse is given by:

$$
\begin{gathered}
\text { Circle } \mapsto \frac{m(m-1)}{2} O\left(N^{3}\right) \\
\text { Ellipse } \mapsto \frac{m(m-1)(m-2)(m-3)}{24} O\left(N^{3}\right)
\end{gathered}
$$

It should be noted that where a parallel implementation is possible then the $O\left(N^{3}\right)$ term will become $O(4 N)$ giving an appreciable speed up in computation time. Using a hypothetical 10MIPS machine as a benchmark, the above expressions were used to calculate the estimated computation times shown in Fig. 1a and 1b. In the case of the ellipse this is shown in seconds and for the circle seconds $\times 10^{-3}$

It can be seen that the feature point density is critical in the case of ellipse detection. Where this is $\simeq 100$ points then the calculation time is $\simeq 48 \mathrm{~s}$. For $70 \mathrm{pts}$ this decreases to $12 \mathrm{~s}$. In all cases it is advantageous to keep the feature point density as low as possible without significantly affecting the accuracy of detection. In the case of a straight line Kiriyati et al[4] have shown that it is possible to use a very small, randomly sampled subset of image points. A sample size as low as $2 \%$ is shown to be effective. The method was tested in the case of ellipse detection and the results are detailed in the next section.

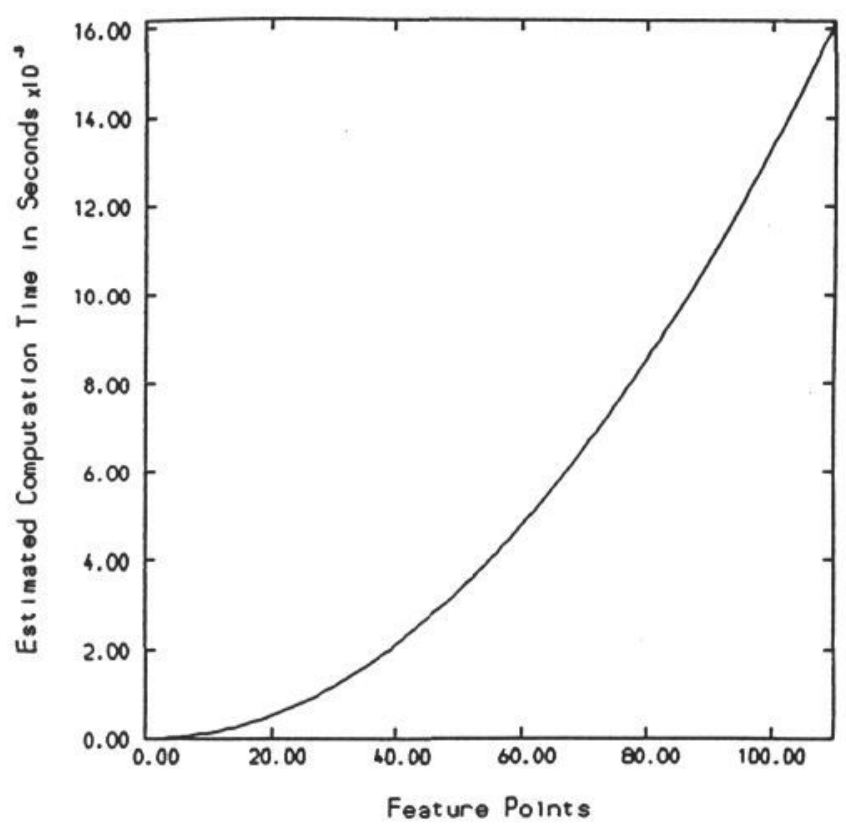

- Fig.1a Circle Estimated Computation Times

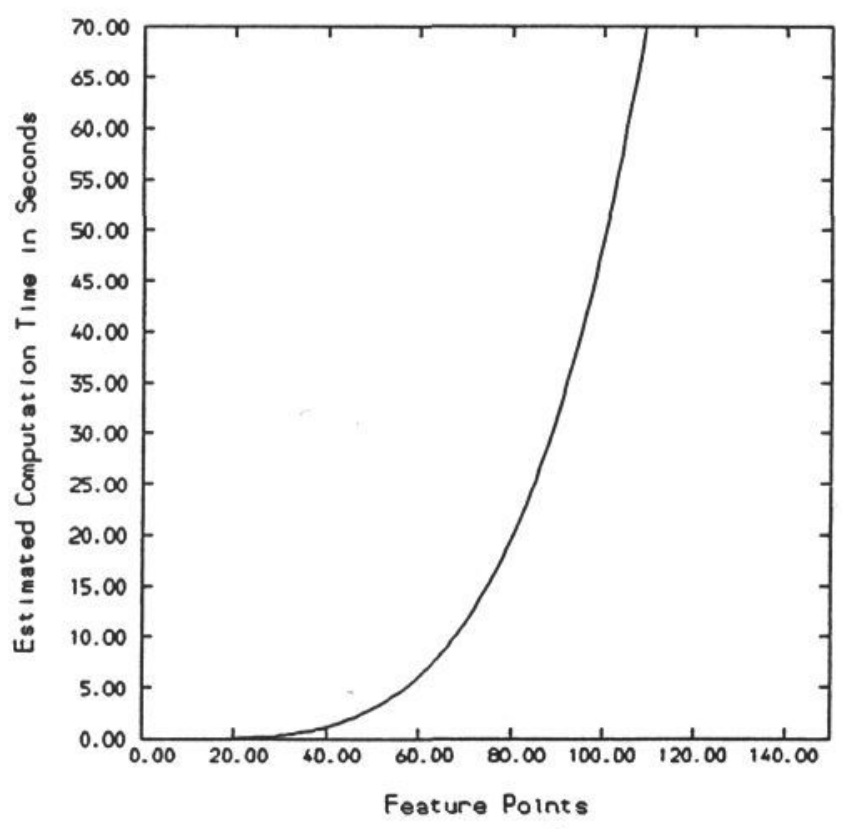

Fig.1b Ellipse Estimated Computation Times

\section{Results}

The algorithm may be activated to detect single features or a mix of features. To date, it is implemented and tested with respect to circle and ellipse detection.

The test image shown in Fig.7 is of a degraded noisy image with examples of multiple circles of differing sizes and some occlusion. The overall signal to noise ratio of the image is 0.64 but because the image was segmented the average signal to noise ratio of each segment was $0.87 \pm 0.08$. 
Fig. 8 shows in mid grey the detected circles and also the segmentation used. The processing has located all of the circles present in the image even those which are partially occluded. It failed to locate the quarter circular arc in the bottom right of the image. The circles were detected with errors in $x_{0}, y_{0}, r_{0}$ of \pm 1 pixel.

Fig. 5 shows the histogram associated with a rejected $(\leq 30 \%)$ estimate of the radius of the circle $\mathbf{A}$ shown in Fig.7. This estimate was the result of transforming the points shown in the segment labelled 1 in Fig. 8. The histogram shows wide multiple peaks. A larger segment size (segment 2, Fig. 8) gave the accepted estimate whose histogram is shown in Fig. 6. Here the peak is well defined and isolated.

As a rough comparison and using weights of 1 for addition and subtraction and 2 for multiplication or division, the test image shown requires only $0.7 \%$ of the calculations necessary to achieve the same resolution of result using a standard Hough transform (SHT) algorithm. Peak detection is only one dimensional and will result in significant computational savings. The opportunity exists to reduce further the run time of the algorithm by using a parallel implementation.

The algorithm was tested without further refinement on the real image of some coins (Fig. 2). The elliptical appearance of the coins is due to non-square pixels. Fig. 3 shows the edge image data and Fig. 4 shows the detected objects and the segmentation used.

The test image used for ellipses is shown in Fig. 9. Four numbered ellipses are shown. Taking a random sample of $30 \%$ of the points in each of the segments associated with an ellipse, the algorithm was able in all cases to estimate the center coordinates to within 2 pixels. These estimates were not bettered by taking a larger sample of points. At this (30\%) sampling rate, the parameters associated with ellipses 3 and 4 were estimated to within 2 pixels for $a$ and $b$ and to within $1^{\circ}$ for $\alpha$. Ellipses 1 and 4 did not have good estimates of $a$ or $b$ until $60 \%$ and $80 \%$ of the points were sampled respectively. The angle of rotation of both ellipses 1 and 4 was poorly determined in all cases. This is to be expected because for $\alpha \neq 0$ or $\frac{\pi}{2}$ the problem becomes ill-conditioned[3]. This suggests that a more computationally efficient formulation of the algorithm would involve a two stage approach as in [5]. The center co-ordinates could be estimated very quickly using only a small sample of the feature points. In general, for $\alpha \simeq 0$ or $\frac{\pi}{2}$ this would be sufficient to estimate all of the parameters with-

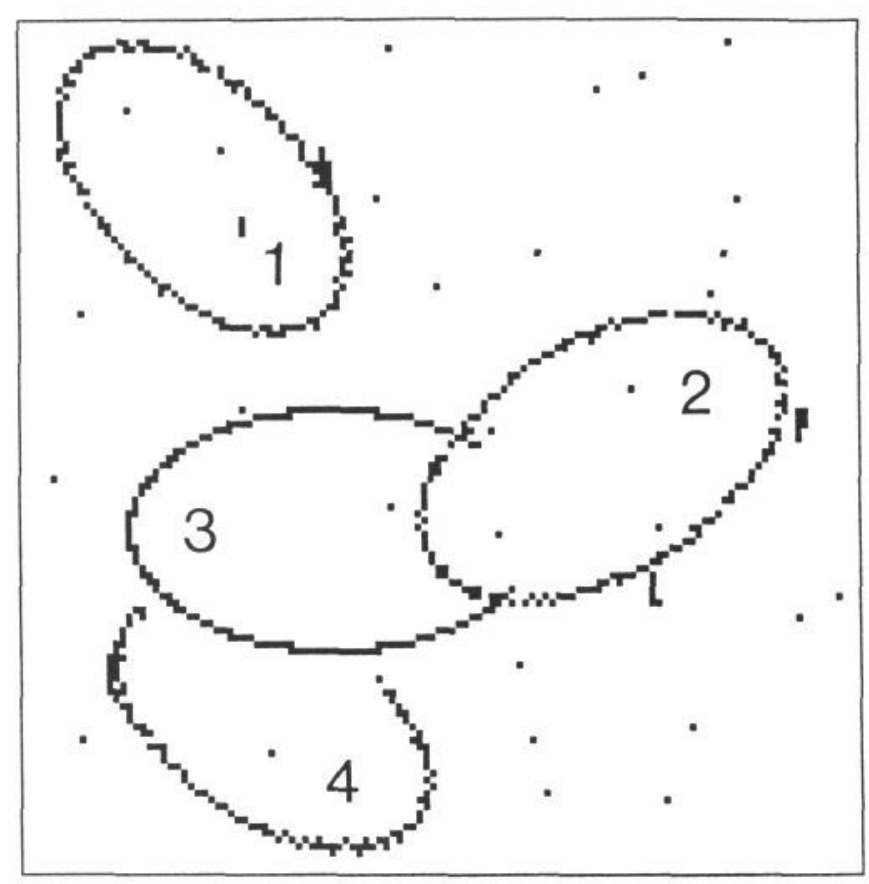

Fig. 9 Ellipse Test Image

out engaging further processing stages. Where $\alpha \neq 0$ or $\frac{\pi}{2}$ then the estimated center co-ordinates can be used to translate the feature points to this new origin and the problem is then reduced to one of the determination of the three remaining parameters with the associated savings in computation time.

\section{5 . Conclusions}

Although much work remains in testing and implementing the algorithm with respect to ellipse detection and mixed feature detection, it has been shown that the present approach offers a significant improvement to previous suggested implementations of the Hough transform. It is computationally less intensive. The proposed method is more efficient in memory utilization. If $T$ is the resolution in the transform space and $n$ is the number of parameters under detection then the SHT requires $T^{n}$ memory allocations in order to maintain a unified $n$ dimensional accumulator. The Dynamic Generalized Hough transform (DGHT) requires only $n T$ memory allocations, each one dimensional accumulator being independent of all other $(n-1)$ accumulators. Using the (DGHT) the parameters can be calculated and accumulated independently. Peak detection is one dimensional and can also be accomplished independently. The method provides an efficient feedback mechanism linking the accumulated boundary point evidence and the contributing boundary point data. 
It achieves this goal with an intelligent monitoring of the transformation re-directing the processing as appropriate to the shape under detection. The method provides much scope for parallel implementations.

\section{References}

[1] Hough P.V.C. Method and means for Recognising complex patterns. U.S. Patent No. 3069654, 1962

[2] Ballard D.H. Generalizing the Hough Transform to detect arbitrary shapes, Pattern Recognition, Vol 13. No. 2, 111-122, 1981.

[3] Forman A.V A modified Hough transform for detecting lines in digital imagery, 151160, SPIE, Vol 635, Applications of Artificial Intelligence III, 1986.

[4] Jain A.N. and Krig D.B. A robust Hough transform technique for machine vision, Proc. Vision 86, Detroit, Michigan, 86.

[5] Leavers V.F. and Sandler M. B. An efficient Radon transform BPRA 4th International Conf., Cambridge, 1988.

[6] Li H., Lavin M.A. and LeMaster R.J. Fast Hough Transform Proc. of 3rd workshop on computer vision: Bellair, Michgan 1985.

[7] Li H. Fast Hough Transform for multidimensional signal processing, IBM Research report, RC 11562, York Town Heights 1985

[8] Illingworth J. and Kittler J. The adaptive Hough transform in press IEEE T-PAMI 1987

[9] R.S. Wallace $A$ modified Hough transform for lines, IEEE conference on computer vision and pattern recognition, San Francisco 1985, 665-667.

[10] Tsukune H. and Goto K. Extracting Elliptical Figures from an Edge Vector Field IEEE Computer Vision and Pattern Recognition Conference, Washington, 1983.

[11] Tsuji and Matsumoto F. Detection of Ellipses by a Modified Hough Transformation, IEEE Trans on Computers, C-27, No. 8, 1978 .

[12] Gerig G. Linking image-space and accumulatorspace. A new approach for object recogni- tion Proc. of First Int. Conf. on Computer Vision, London, June 1987

[13] Yuen H.K, Illingworth J and Kittler J. , Ellipse Detection Using the Hough Transform, Alvey Vision Conference, Manchester, 1988.

[14] Leavers V.F. Dynamic Generalized Hough Transform, British Patent Application, April 1989.

[15] Leavers V.F., Ben-Tzvi D. and Sandler M.B. A Dynamic Hough Transform for Lines and Circles, Alvey Vision Conference, Reading, 1989

[16] Leavers V.F. The Dynamic Generalized Hough Transform, First ECCV conf., Antibes, France, April 1990.

[17] Deans S.R. Hough transform from the Radon transform. IEEE Trans. Pattern Analysis and Machine Intelligence. Vol. PAMI-3, No., March 1981.

[18] Gel'fand I.M., Graev M.I. and Vilenkin N.Ya. Generalized functions Vol 5, Academic Press, New York, 1966.

[19] A.B. Forbes, Fitting an Ellipse to Data, National Physical Laboratory Report, DITC 95/87 - December 1987.

[20] Kiriyati N., Eldar Y. and Bruckstein A. A Probabilistic Hough Transform, 6th Israeli Conference on Artificial Intelligence, Vision and Pattern Recognition, Tel Aviv 1989. 


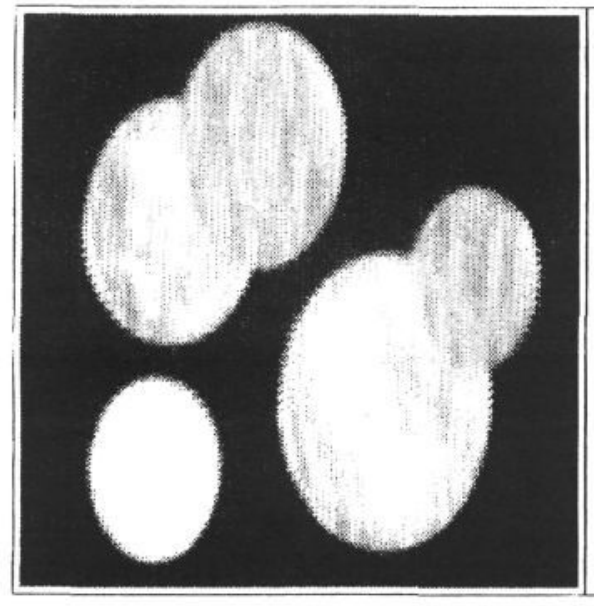

Fig.2 Image of Coins

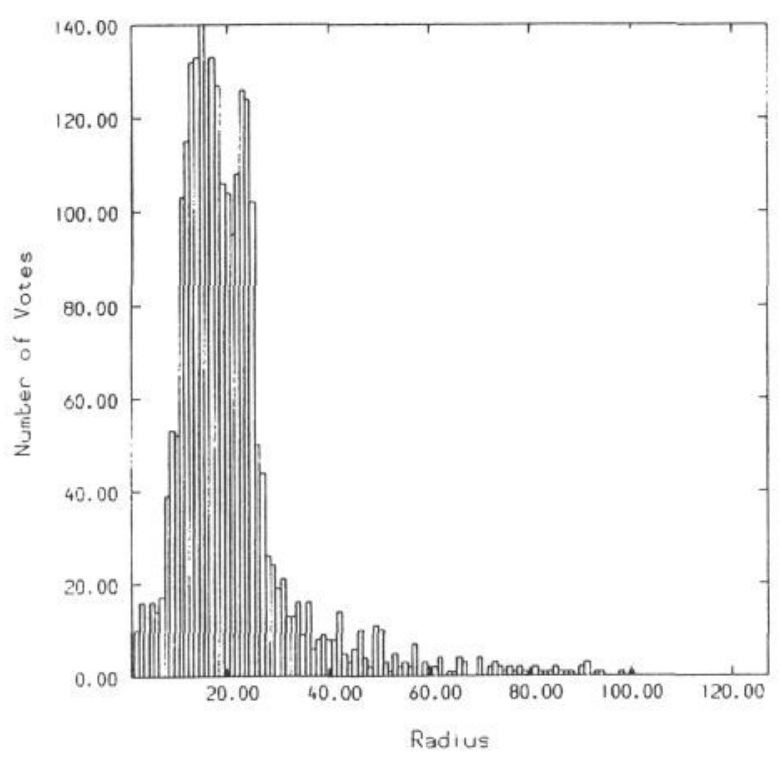

Fig.5 Rejected Estimate

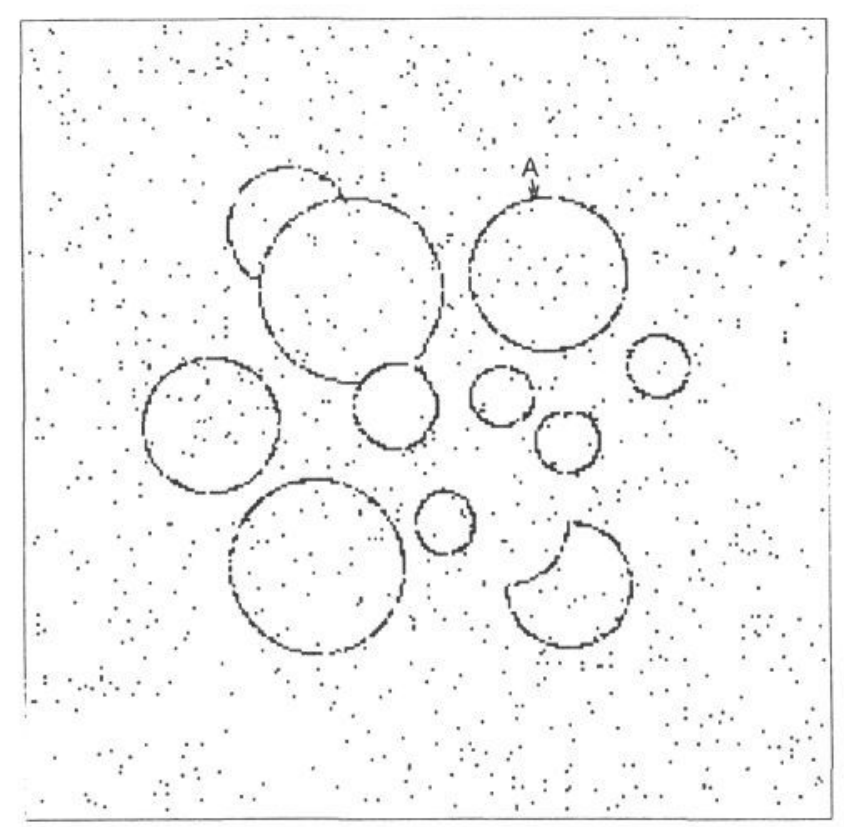

Fig.7 Degraded Test Image

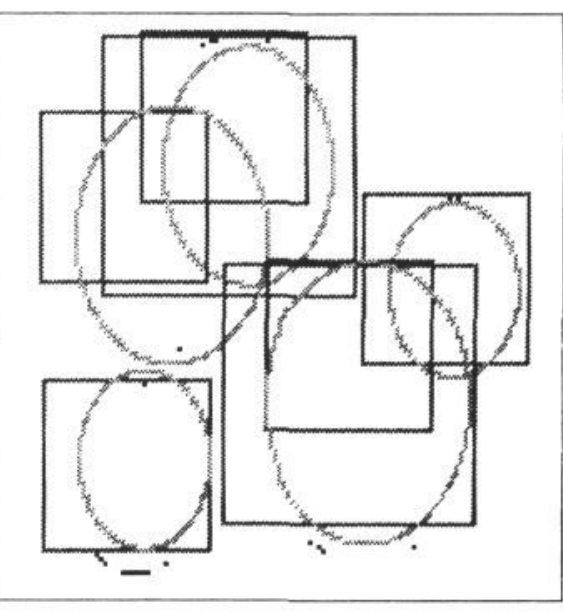

Fig.4 Detected Objects

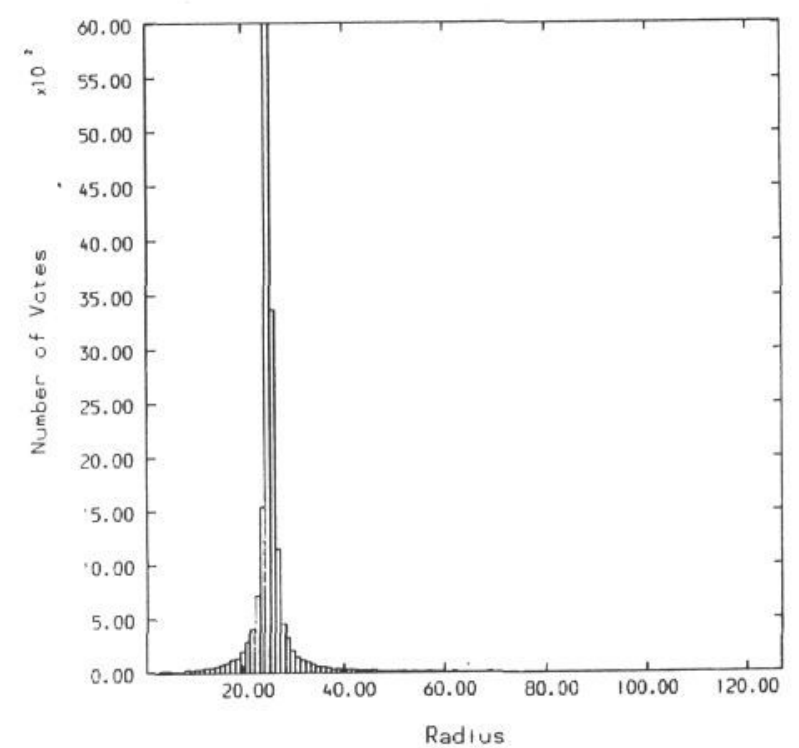

Fig.6 Accepted Estimate

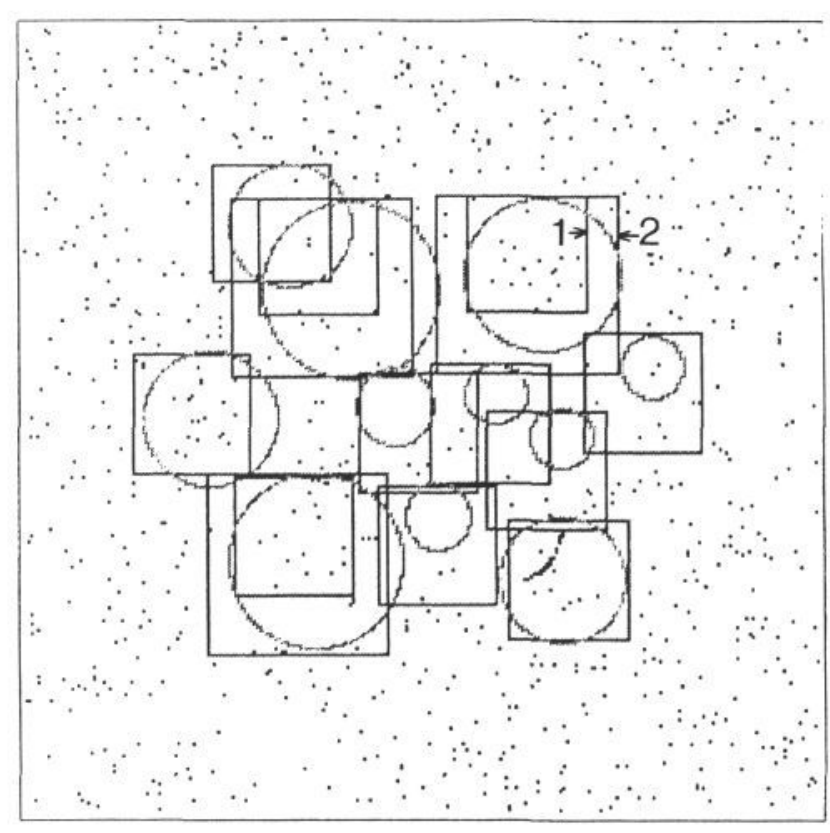

Fig.8 Detected Circles 